\title{
Finding the location of the instantaneous center of rotation using a particle image velocimetry algorithm
}

Tom Claessens

Citation: American Journal of Physics 85, 185 (2017); doi: 10.1119/1.4973427

View online: http://dx.doi.org/10.1119/1.4973427

View Table of Contents: http://aapt.scitation.org/toc/ajp/85/3

Published by the American Association of Physics Teachers

\section{Articles you may be interested in}

Why trains stay on tracks

American Journal of Physics 85, (2017); 10.1119/1.4973370

New angles on the reverse sprinkler: Reconciling theory and experiment

American Journal of Physics 85, (2017); 10.1119/1.4973374

Project-based physics labs using low-cost open-source hardware

American Journal of Physics 85, (2017); 10.1119/1.4972043

Metamaterial hyperlens demonstration of propagation without diffraction

American Journal of Physics 85, (2017); 10.1119/1.4972243

\section{Physics from Planet Earth}

A re-structured and re-imagined textbook for the introductory calculus - based mechanics course Discover a distinctly different approach that is sure to captivate your students!

See: https://www.crcpress.com/9781439867839

and https://physicsfromplanetearth.wordpress.com

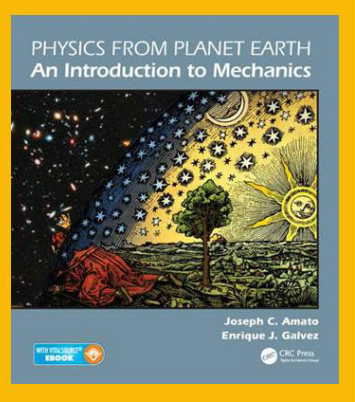




\title{
Finding the location of the instantaneous center of rotation using a particle image velocimetry algorithm
}

\author{
Tom Claessens \\ Department of Materials Science and Engineering, Faculty of Engineering and Architecture, Ghent University, \\ Ghent 9000, Belgium
}

(Received 8 April 2016; accepted 13 December 2016)

\begin{abstract}
This work is about planar rigid-body kinematics and, in particular, the principle of the instantaneous center of rotation (IC). Using a computer simulated approach, a workflow is presented that results in a visual representation of the locus of the IC, based on particle image velocimetry (PIV). Here, a small number of digital animations of textured objects are created with multibody dynamics software, and later imported in PIV software to extract the velocity field (magnitude and direction) of objects moving within a plane. We believe the workflow presented may help learners improve their understanding of the concept of the IC, thus enhancing their knowledge of rigid body kinematics. (c) 2017 American Association of Physics Teachers.

[http://dx.doi.org/10.1119/1.4973427]
\end{abstract}

\section{INTRODUCTION}

Engineering dynamics is one of the core courses taken by virtually all civil, mechanical, and biomedical engineering students. It is a high-impact course that treats topics of fundamental importance in advanced engineering courses, such as strength of materials, machine design, vibration, structural mechanics, and biomechanics. Typically, this course covers the dynamics of particles, systems of particles, and rigid bodies in one, two, or three dimensions. Two-dimensional rigid body kinematics, which focuses purely on the motion of non-deformable bodies undergoing plane motion (without considering the effect of forces), is regarded as one of the more complex topics within this field. To fully understand this topic, students have to familiarize themselves with a number of essential concepts, including (relative) position and displacement as well as linear and angular velocity and acceleration of a body, usually with vector algebra formulations. Rigid body kinematics forms an essential basis for rigid body dynamics, in which the relation between forces and motion is being investigated.

General plane motion, in which the body undergoes simultaneous translation and rotation, is covered in virtually all introductory mechanical engineering textbooks. ${ }^{1-4}$ In physics textbooks, this topic is often treated less theoretically via a number of rolling and/or slipping cylinder problems. ${ }^{5-7}$

From theory and examples, it can be shown that any plane motion of a body can be considered as a superposition of a translation, defined by the motion of an arbitrary reference point $A$, and a simultaneous rotation about $A$ (see Fig. 1). This property results in a fundamental kinematic vector equation based on relative motion given by

$$
\vec{v}_{B}=\vec{v}_{A}+\vec{v}_{B / A}=\vec{v}_{A}+\vec{\omega} \times \vec{r}_{B / A} .
$$

This two-dimensional vector equation expresses the relation between the velocities of two chosen points $A$ and $B\left(\vec{v}_{A}\right.$ and $\left.\vec{v}_{B}\right)$, the position of one point relative to the other $\left(\vec{r}_{B / A}\right)$, and the angular velocity of that body $(\vec{\omega})$, perpendicular to the plane of motion. In practice, Eq. (1) contains five unknowns (assuming that $\vec{r}_{B / A}$ can be easily derived from the figure in the textbook problem) and is commonly used to solve standard textbooks problems about rigid body kinematics, such as the rolling cylinder (with or without slipping), gear systems, or piston crankshaft mechanisms. The principle of superposition and Eq. (1) are illustrated in Fig. 1 (based on Ref. 1), showing the kinematics of a rod sliding down a wall.

While the velocity and position vectors in Eq. (1) are relatively easy to interpret, the angular velocity vector $\vec{\omega}$ is conceptually more challenging for students to grasp. Indeed, in contrast to velocity vectors $\vec{v}_{A}$ and $\vec{v}_{B}$, vector $\vec{\omega}$ is rather a symbolic (free) vector along the axis of rotation and with a magnitude equal to the angular velocity. The fact that in reality the body does not move along $\vec{\omega}$ was recently highlighted by Demircioglu et al. ${ }^{8}$ These authors attempted to demonstrate the physical meaning of $\vec{\omega}$ (albeit in case of a rotation about a fixed axis) using the observable motion of a screw mounted to a remotely operated toy car.

Another useful and closely related topic found in the majority of the engineering mechanics textbooks is the instantaneous center of rotation, also known as the instantaneous center of zero velocity, or briefly the instant center (IC). The IC is a point in the plane of motion whose velocity at the instant under consideration equals zero $\left(\vec{v}_{\mathrm{IC}}=\overrightarrow{0}\right)$. As a result, every other point on the rigid body rotates instantaneously about a (virtual) axis through the IC (which lies either on or outside the body) at the instant under consideration. In textbook problems, one of the challenges is typically to find such a point with zero instantaneous velocity. When successful, the student can apply an equation from

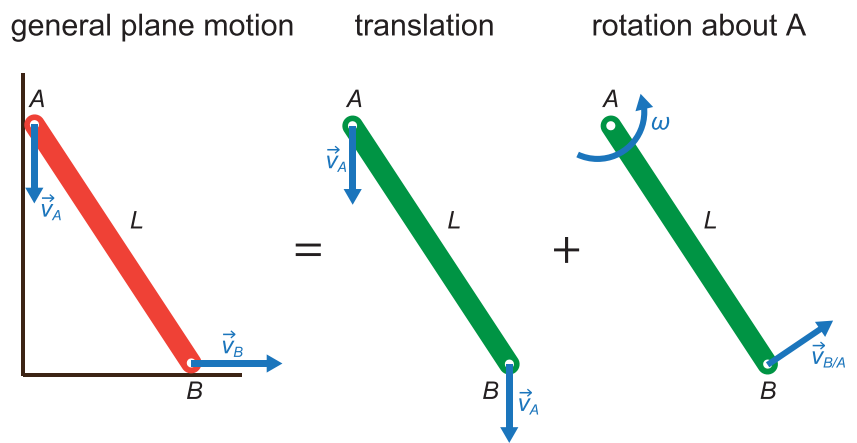

Fig. 1. General plane motion of a body is composed of translation and rotation about a fixed point. Equation (1) is derived from the vectors shown at point $B$ of the rod. 
(a)

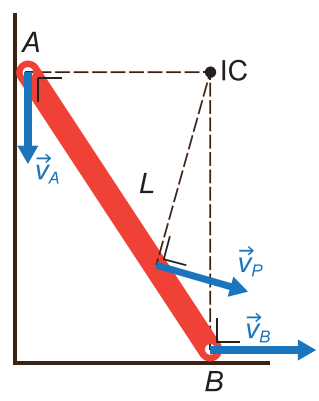

(b)

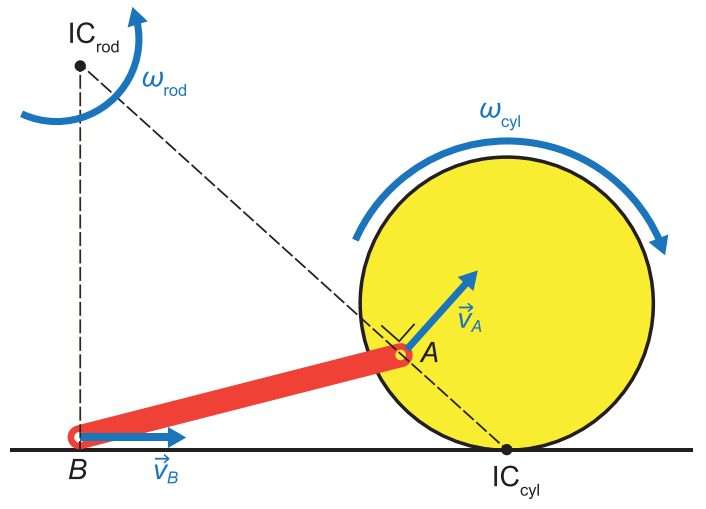

Fig. 2. (a) Instant center (IC) of the rod in the given position. (b) Different IC's of the different bodies of a mechanism, consisting of a wheel dragging a rod.

pure rotation to find the velocity vector of any other point $P$ on the body as

$$
\vec{v}_{P}=\vec{\omega} \times \vec{r}_{\mathrm{P} / I C}
$$

with $\vec{r}_{P / \text { IC }}$ being the position of point $P$ relative to the instant center IC (see Fig. 2).

Depending on the type of problem, several approaches can be followed to determine the location of the IC, which are all based on basic theory about circular motion. First, (known) velocities of a point on the body must be perpendicular to the position vector of that point relative to the IC. Second, the magnitudes of the velocity vectors are linearly related to the distance from the IC. If applied well, the IC approach is a very efficient and quick technique to solve elementary kinematics problems involving linear and angular velocity vectors, though it often requires some additional trigonometry to be performed. In the Appendix, two solutions to an introductory kinematics problem are presented (one using the vector equation and one using the IC concept).

This particular topic in rigid body kinematics was chosen based on the author's experience as a mechanics teacher of first year engineering students (around 500 students each academic year). Despite the apparent simplicity of the IC concept, I have observed that it seems far less "popular" among mechanical engineering students. When students were allowed to use their preferred method, the IC concept was commonly avoided in favor of the vector analysis method of Eq. (1). Moreover, if students were required to work with the IC concept, problems were often solved incorrectly. The fact that more than $75 \%$ of the students provided an incorrect answer to the question on what is the position of the IC of the rod in Fig. 2(b) (adapted from Ref. 1) shows that there may be a number of misconceptions about rigid body kinematics, and the application of the IC method in particular. The reasons behind this remain largely unknown.

For many students, visualizing the motion of a mechanical system, usually represented as a static image in a textbook or on the blackboard, is challenging. Although both computer simulation and animation and physical models of simple mechanisms have proven very effective in this sense, neither of them actually demonstrates the location of the IC in a straightforward manner. Apart from a very common photo of the contact point between a bicycle wheel rolling without slipping and the ground, ${ }^{2}$ we are not aware of any other educational, physically demonstrable tool for demonstrating the existence of the IC (which is not a physical point).
The central question is this work is how one can shed more light on the IC method and whether a relatively simple tool or technique can be presented to deal with this issue. We believe that the IC concept can be reinforced if it could be located it in a straightforward and visual way.

\section{AIM}

In this paper, we demonstrate an innovative approach to directly measure and locate the instant center of velocity of a rigid body undergoing general plane motion. The method uses an algorithm used in Particle Image Velocimetry (PIV), which is an optical non-intrusive technique for investigating flowing fluids (gases and liquids). The broader purpose of this work is twofold. First, we hope to increase introductory engineering and physics students' understanding of planar rigid body kinematics, and the instantaneous center of rotation in particular. The IC is found by providing a graphical representation of the velocity vector field of a rigid body undergoing general plane motion. The vector field should clearly indicate a point with zero velocity, the instantaneous center of rotation. Second, the workflow presented tends to be an example of a creative and unusual way to analyze rigid body motion by means of an advanced experimental technique for flow studies, which is used outside its traditional application area. It is expected that this could enhance the students' (and teachers') interest in nonstandard technologies early in their education. To the best of the author's knowledge, no other study on planar rigid body kinematics has been performed previously with a PIV-based approach.

Note that the aim of this work is not to develop a novel procedure to replace the current solution techniques outlined in standard textbooks; it merely provides complementary graphical information that should help students gain a better qualitative understanding of the IC concept.

\section{METHODS AND WORKFLOW}

Basically, the workflow is rather straightforward and consists of image creation and image processing (see Fig. 3).

\section{A. Image creation}

A small set of animations was created as follows. CAD models of rigid bodies were generated in the commercial software $\mathrm{NX}^{9}$ and subsequently imported in a physics-based simulation software package Simwise $4 \mathrm{D}^{10}$ (formerly known as Visual Nastran 4D and Working Model 3D). This software is 


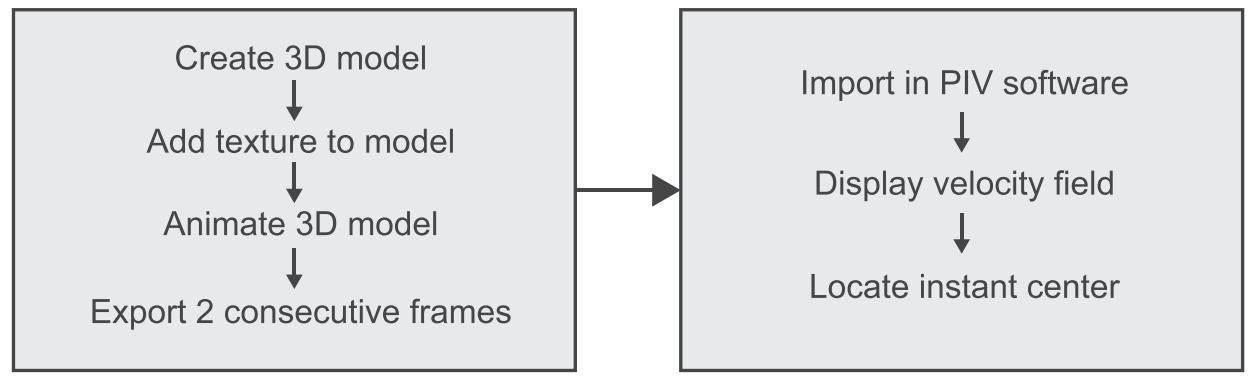

Fig. 3. Digital workflow, showing all steps that were taken to create the images shown in Fig. 7, starting from a 3D model of an object (rigid body). Note: the left panel can be replaced with a physical counterpart, where images of physical rigid bodies textured with printed particles are recorded using a digital camera.

highly specialized in simulating kinematics and dynamics of rigid bodies. Motion simulations are performed by prescribing the motion of the bodies, or by imposing loads. To allow for a PIV analysis, a surface texture with "virtual" particles was mapped to the models. The texture was a $300 \times 300$ pixel bitmap with randomly distributed black dots on a white background. The size of the dots was around 5 pixels.

The result of the motion simulations can then be exported, either with or without the added particles, as a movie or as consecutive still frames. The inter-frame time interval (temporal resolution) can be chosen by the user, depending on the specific example. For the animations shown, the temporal resolution varied between 10 and $20 \mathrm{~ms}$. In this study, two consecutive frames from the motion simulations were saved in uncompressed TIFF format to retain the quality of each of the separate images.

\section{B. Image post-processing: Displaying the velocity field by means of PIV}

Digital PIV is currently the preferred technique for measuring full field velocities in flowing fluids (gases and liquids) in a quasi-non-intrusive way. A standard implementation permits measurement of two velocity components within a plane, while high-end hardware configurations (holographic and tomographic PIV) allow acquisition of three velocity components within a three-dimensional volume at very high temporal resolutions (up to $10,000 \mathrm{~Hz}$ ). PIV has widespread use in the fields of aerodynamics, bio-fluid flows (e.g., artificial heart valves), combustion processes, river hydraulics, and even soil motion. Often, PIV is used to validate the results of computer simulations of complex flow problems. The method requires seeding the fluid under investigation with small (on the order of $\mu \mathrm{m}$ ) particles, which are believed to faithfully follow the fluid flow. One or more digital cameras record the particle images, and post-processing software is used to analyze consecutive particle images and to deliver information about the vector velocity field. The fundamental principle of PIV as a velocity measurement technique is very closely related to the definition of (average) velocity

$$
\vec{v}=\frac{\Delta \vec{r}}{\Delta t},
$$

with $\Delta \vec{r}$ being the displacement of the particles and $\Delta t$ being the time interval between two successive frames. The particle images are divided into small interrogation regions (typically with a dimension of $64 \times 64$ pixels). Cross-correlation techniques are then used to find the displacement $\Delta \vec{r}$ of these interrogation regions between two consecutive frames of the animation. In the end, the particle image velocity algorithm yields a full-field color-coded visualization of velocity magnitude and direction.

An example of a typical PIV experiment, in bio-fluid mechanics, is shown in Fig. 4. Interested readers are referred to Refs. 11-14 to gain in-depth information about the hardware and underlying algorithms used in PIV.

In the current study, only the second part of a traditional PIV workflow is followed, i.e., only the digital images exported from the motion simulations were used as input. The motion of the (virtual) particles was investigated using PIVlab $^{15}$ (Fig. 5), a free, open-source software program implemented in MATLAB. ${ }^{16}$ The region of interest is chosen by the user, and encloses the body of interest as closely as possible. Several consecutive frames from the animation can be imported and used to derive velocity information. The (a)

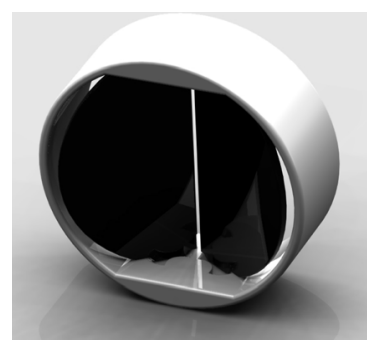

(b)

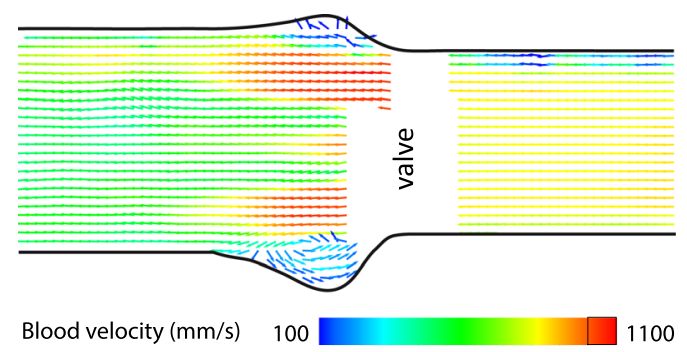

Fig. 4. Representative results of a typical PIV experiment in biofluid mechanics. (a) Rendered CAD model of a bi-leaflet mechanical heart valve. (b) Full field visualization of the flow velocity vectors in the neighborhood of this valve. Measurements were performed in a hydraulic in vitro model with a blood mimicking fluid. Reproduced with permission from S. Annerel et al., Med. Eng. Phys. 36(8), 1014-1023 (2014). Copyright 2014, Elsevier (Ref. 31). For clarity, some labels were digitally added. 


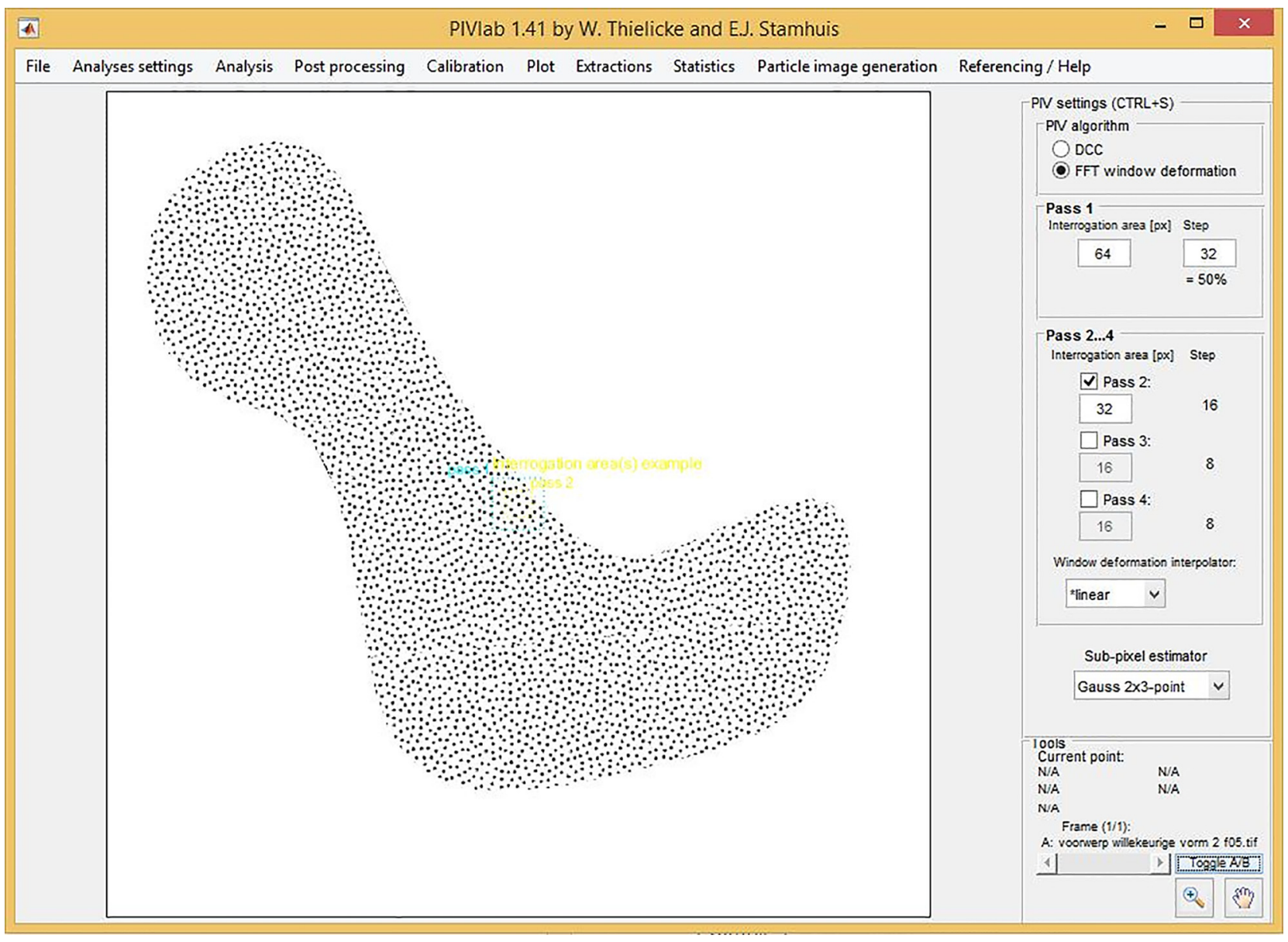

Fig. 5. Screenshot taken from the PIV postprocessing software program PIVlab (Ref. 15). A frontal view of one of the bodies used in the simulations is shown.

final velocity vector field was obtained in several consecutive steps with decreasing grid size (starting at $64 \times 64$ and moving to $32 \times 32$ pixels). On a standard laptop (Intel Core $^{\mathrm{TM}} 2$ Duo Processor P8600), the analysis takes about $3 \mathrm{~s}$. Irrelevant velocity vectors outside the region of interest (the rigid body itself) were removed manually after the analysis to improve the clarity of the images. Spatial calibration was easy to perform since the relation between the number of pixels in the image and the size of the rigid body is known. Temporal calibration data were derived from the time interval between the various frames in the simulation (as defined earlier by the user in Simwise 4D). The point with zero velocity represents the instant center of rotation.

\section{TEXTBOOK EXAMPLES}

Two CAD models (created in NX and then imported in Simwise 4D for texturing) were used to illustrate the presented workflow (see Fig. 6). One is a circular disk of radius $2 \mathrm{~m}$. The second model is a rigid body with an arbitrary shape that has both a width and height of about $2 \mathrm{~m}$. The
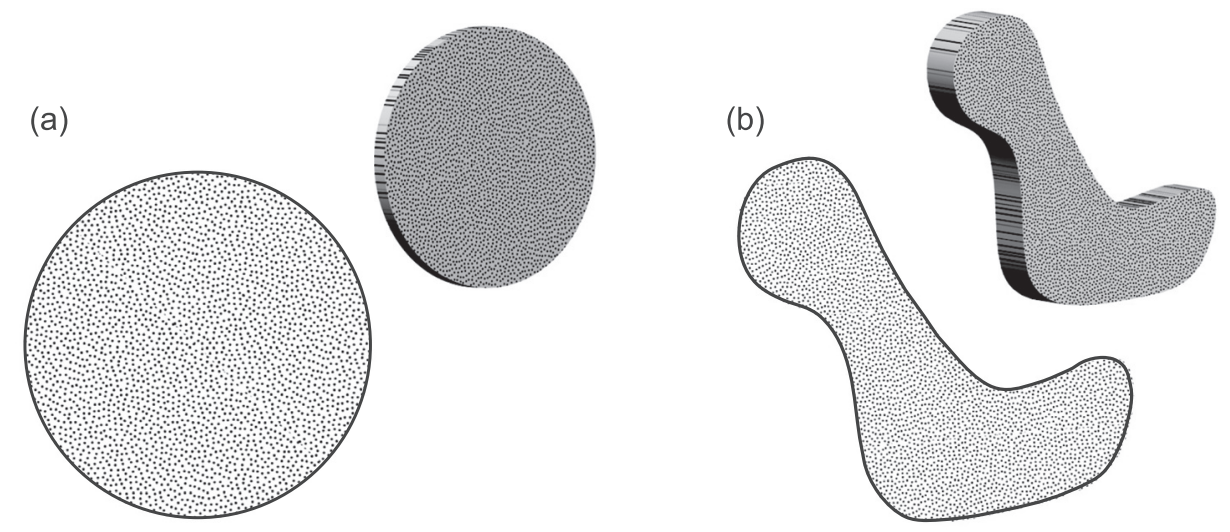

Fig. 6. Two textured cad models: (a) circular disk and (b) body with arbitrary shape. 
examples shown are based on generic textbook problems. ${ }^{1-4}$ The kinematics of the bodies was prescribed in Simwise 4D.

\section{A. A rolling disk}

Example 1 [Fig. 7(a)] shows a very common situation where a homogeneous disk rolls to the right without slipping on a flat surface. The angular velocity was set to $1 \mathrm{rad} / \mathrm{s}$. The maximum velocity of $4 \mathrm{~m} / \mathrm{s}$ is obtained at the top of the disk. As anticipated, the instant center with zero velocity is at the contact point with the flat surface. To some extent, the technique applied is similar to a photograph taken from a rolling wheel without slipping. ${ }^{2}$ However, in the latter case no quantitative information is given for the velocity field.

\section{B. A disk between two flat surfaces}

The next example [Fig. 7(b)] shows a disk that moves without slipping between two flat surfaces (one at the top and one at the bottom) moving with different velocities. This configuration is similar to a dual rack and pinion configuration. The points of contact move with the same velocity as the two plates. The image clearly shows that at the given
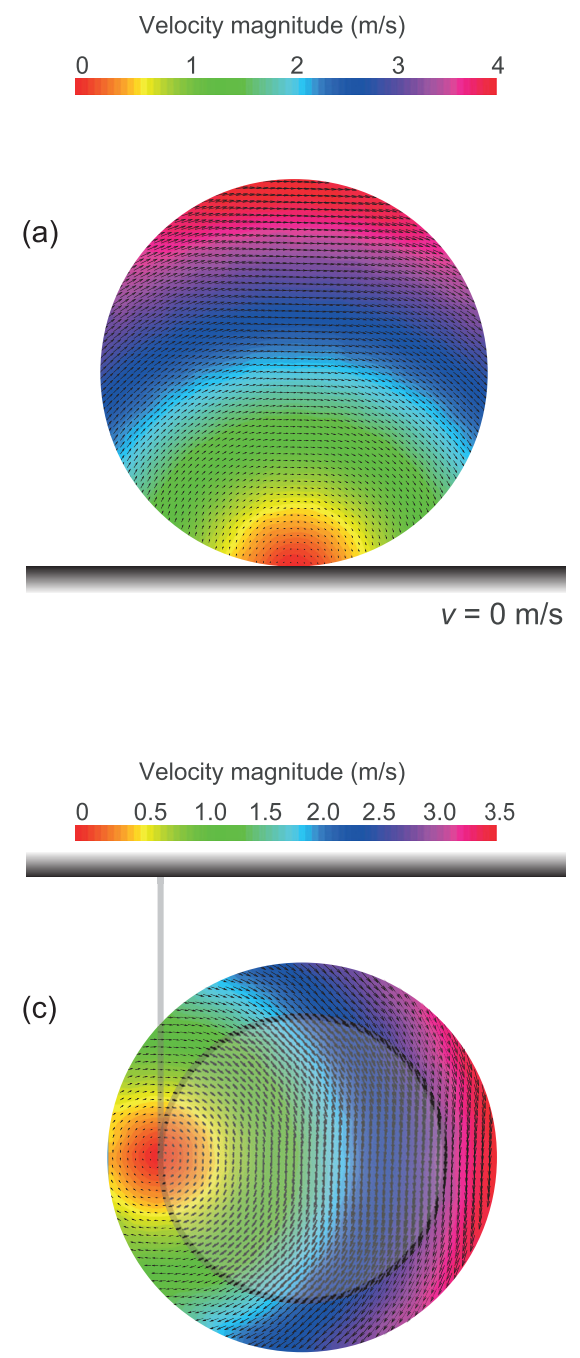

instant the disk rotates around a point in the lower half of the disk. From the two known velocities, it can be easily found analytically that the IC is located at exactly one third of the height (diameter) of the disk.

\section{Unwinding spool}

The third example [Fig. 7(c)] demonstrates the velocity field of a vertically falling spool that unwinds the rope with an angular velocity of $1 \mathrm{rad} / \mathrm{s}$. The spool has an inner radius of $1.5 \mathrm{~m}$ and an outer radius of $2 \mathrm{~m}$. Similar to the rolling disk, the IC lies on the point of contact between the rope and the small disk. The velocity of the rightmost point located on the outer rim of the spool equals $3.5 \mathrm{~m} / \mathrm{s}$.

\section{Body with arbitrary shape moving between two slots}

The last example [Fig. 7(d)] shows a rigid body with an arbitrary shape that is confined to move in a horizontal and a vertical slot. The angular velocity was $1 \mathrm{rad} / \mathrm{s}$. This problem is comparable to a sliding ladder problem. In this particular condition, the IC falls outside of the body. Without

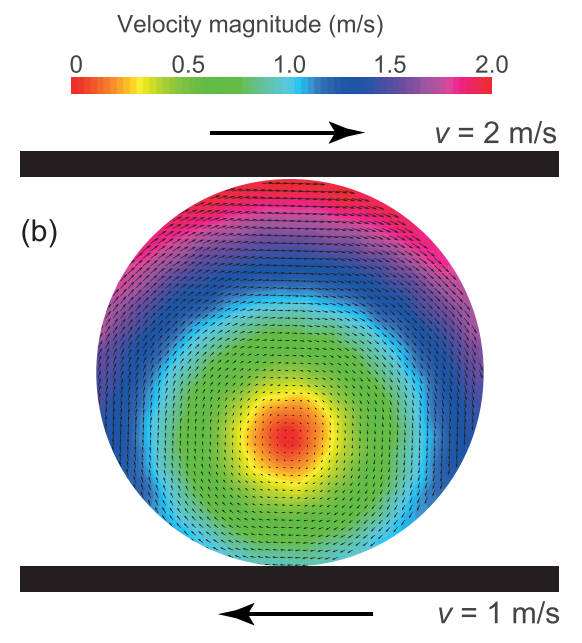

(d)

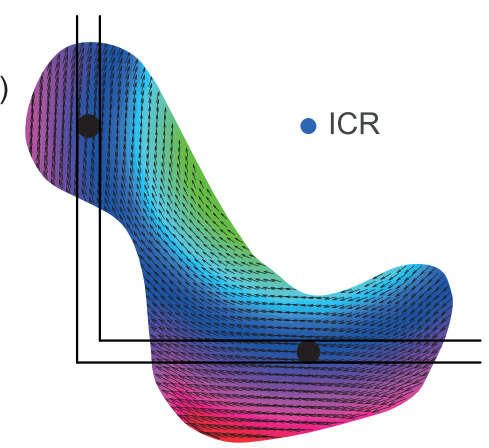

Fig. 7. Vector velocity field with velocity magnitude represented by shading (color online): (a) rolling disk (or cylinder); (b) disk (or cylinder) between two plates with different velocities; (c) unwinding spool; and (d) body with arbitrary shape moving between two slots. 
performing any calculations, the image already gives a clear impression of where the IC lies.

\section{DISCUSSION}

This paper is limited to planar kinematics of rigid bodies, known as quite a challenging topic in most introductory mechanical engineering and physics courses. The first three examples shown are rolling cylinder problems, which are difficult to solve for students according to recent research. ${ }^{17-19}$

Equation (1) constitutes a basic relation between two velocities of random points $A$ and $B$ on a body, and is frequently applied to solve general introductory textbooks problems in plane kinematics. Its practical usefulness can be demonstrated with "real life" problems such as a wheel rolling with or without slipping, a ladder sliding down a wall and the well-known piston-crankshaft movement. The theory behind this equation is traditionally taught during a classroom-based lecture, using a number of (static) schematic blackboard drawings with linear and angular velocity and position vectors at discrete locations. Vector equations are converted to scalar equations before working out the solution of the textbook problems. Common difficulties encountered by students include the (abstract) vector representation of angular velocity, ${ }^{8}$ and their lack of thorough insight into the motion (and degrees of freedom) of the moving bodies.

A concept closely related to the vector velocity equation is the instant center of rotation. ${ }^{1-4}$ The instantaneous center of rotation (IC) of a rigid body undergoing general plane motion is a point on (or outside) the body, which has an instantaneous velocity equal to zero. As such, it appears that the body is instantaneously rotating about a fixed axis through the IC, and any other point $P$ follows a circular path around the IC. The velocity of point $P$ can be readily found by rewriting Eq. (1) as

$$
\vec{v}_{P}=\vec{v}_{\mathrm{IC}}+\vec{v}_{P / \mathrm{IC}}=\vec{v}_{P / \mathrm{IC}}=\vec{\omega} \times \vec{r}_{P / \mathrm{IC}} .
$$

From an academic viewpoint, this concept is commonly proposed as a valid and often time-saving alternative technique for solving planar kinematics problems. Apart from that, the IC concept also has many practical purposes. In musculoskeletal biomechanics, for example, the concept has previously been used to investigate cervical spine movement, ${ }^{20,21}$ to examine gender differences in knee kinematics, ${ }^{22,23}$ and to estimate the effect of a surgical intervention of the shoulder. ${ }^{24}$ In mechanical engineering, it has proven useful for studying complex link mechanisms, ${ }^{25}$ the roll behavior of a car, ${ }^{26}$ and it can help in designing vehicle suspensions systems ${ }^{27}$ or linkage system for trailers. ${ }^{28}$ Figure 8 demonstrates the use of the IC in biomechanics. The position of two identifiable points $A$ and $B$ are marked on the femur and then traced during flexion. The points between successive image frames are joined and the perpendicular bisector is drawn. The IC is given by the intersection of the perpendicular bisectors. The path followed by the IC can then be used to evaluate the functioning of the knee joint. $^{29}$

While the IC method is purely based on common sense, and technically sound and robust, it may not seem so straightforward to a learner. Finding the IC indeed requires a kind of reverse engineering to be applied, as the intersection needs to be found after constructing two perpendiculars to the two known velocity vectors of two points. In general plane motion, however, a great number of examples can be shown

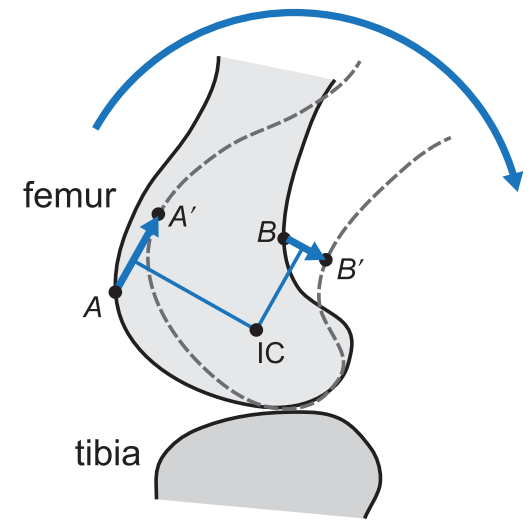

Fig. 8. Instantaneous center of rotation of the femur (thighbone). The points $A$ and $B$ displace to $A^{\prime}$ and $B^{\prime}$, respectively, during the femoral motion shown. Their displacements are represented by the lines $A A^{\prime}$ and $B B^{\prime}$. Perpendicular bisectors of these lines intersect at the instant center of motion (IC) for the displacement shown. The image clearly shows that the IC does not lie on the articular surface. Therefore, unlike a bicycle wheel rotating without slipping, articular surfaces will be sliding on each other (Ref. 29) A similar animation can be found online (Ref. 32). Note that in this particular application, the found instant center of rotation is not instantaneous, but time-averaged.

where naive ideas dominate over theoretical concepts, leading to incorrect answers. As a consequence, we observed that a significant percentage of students tend to avoid this method and prefer using Eq. (1) whenever possible, perhaps because of the poor insight in planar rigid body kinematics. Note also that the IC concept is generally not treated in introductory physics textbooks, given that these books cover only circular motion of a particle and rigid body rotation about a fixed axis.

Teaching and learning processes in engineering dynamics can be greatly enhanced by integrating digital animation and simulation tools into the classroom. Today, standalone multibody dynamics software, such as Adams (MSC Software), and most modern engineering design software environments, including Inventor (Autodesk), Solidworks (Dassault Systèmes), Simwise 4D (Design Simulation Technologies), and NX (Siemens PLM Software), allow users to simulate the motion of complex mechanisms consisting of rigid and even flexible bodies. These programs allow an instructor or (advanced) student to create sophisticated visualizations of mechanical systems that are virtually impossible to describe in purely text-based form, and to retrieve information about kinematic and dynamics parameters of interest. These tools are expected to improve traditional teaching and learning activities because they allow for deeper exploration of more complex real-world problems of which the solution cannot be readily found with pencil and paper (and calculator) by the average student. Students are given an opportunity to participate in active learning since more time is left for analysis and interpretation of the results.

In the present work, a user-friendly simulation and animation tool, Simwise 4D, was used to visualize the motion of a small set of simple, but real-life standard kinematics problems. A method was presented to extract still images from a kinematics animation and display the instantaneous velocity vector field. Such alternative types of images (and eventually accompanying animations) contain the maximum amount of information available about motion and are expected to facilitate the learners' comprehension of planar rigid-body kinematics, and in particular the concept of the instant center of 
rotation. We are not aware of any other (CAD) software tool that provides similar educational advantages.

\section{STUDY LIMITATIONS}

Despite its potential value in introductory dynamics education, the proposed strategy may still contain a number of shortcomings. In particular, if the IC changes its position in space as well as on the body while the body is moving within the plane, limitations of the PIV software make it impossible to automatically show the space centrode (the locus of the IC in space) and the body centrode (the locus of the IC on the body).

From a pedagogical point of view, the method is particularly useful if the IC lies within the body. Because the velocity field can only be displayed on top of the body, there is no direct way to locate the IC if it lies outside the body. As such, the process is less appropriate for elongated bodies such as a rod or any other body for which the rotational component is less pronounced than its translational component [see Figs. 2 and 7(d)].

A detailed study on the relation between imaging properties (such as particle size, density, and distribution) and the accuracy of the calculated velocity field has not been performed. Such analyses were considered beyond the scope of this work. However, this step is critical in actual particle image velocimetry measurements in complex fluid flows with high velocity gradients.

For various reasons, the method described here should not be considered as an alternative to the current solution strategies followed in standard mechanics textbooks, mainly because there are too many relatively complex and timeconsuming steps required to obtain the velocity field (e.g., animating the motion of a rigid body, applying the PIV algorithm to obtain the velocity field, etc.).

We are well aware of alternative software solutions such as MATLAB (together with Simscape Multibody, The Mathworks), MapleSim (Maple Software) or System Modeler (Wolfram Research) that offer the potential to design a comparable workflow and provide similar end results in terms of visualization. Also, freeware tools such as Algodoo ${ }^{30}$ could potentially be used to replace Simwise 4D for creating physicsbased animations. For educational purposes, however, we tend to prefer the current approach because of the userfriendliness of the multibody software tool to create animations. In addition, less (behind the scenes) programming is ultimately required compared to the above-mentioned common engineering software environments, which is ultimately beneficial for the instructor as well as for the student.

\section{SUGGESTIONS FOR FUTURE WORK}

In the near future, we foresee switching from the virtual image creation (represented in the left panel of Fig. 3) to a real physical environment where physical mechanisms are made from plate structures (e.g., manufactured from cardboard or wood using laser cutting or CNC machining). After adding a printed texture to the plate, their motion can be analyzed using a sensitive camera with sufficient frame rate (either high-speed or a double frame camera). Similar to an actual PIV analysis the recorded images would then serve as an input for PIV analysis, after which the motion of the particles is to be analyzed and a velocity field created. Being able to quantify and visualize the velocity field of the various components of a selffabricated mechanical system would greatly improve students' insight into rigid body kinematics. Such an approach could encourage advanced students to develop small mechanisms in a lab setting and actually see the different types of planar motion (translation, rotation, and general plane motion), and relate their experimental observations with theory. Also, the data could also be used in physics lectures and demonstrations. It is our intention here to explore such ideas further and to investigate which examples (either virtual or physical) are most suitable for teaching planar motion.

\section{CONCLUSIONS}

In this study, we briefly reviewed the basic concepts of planar rigid-body mechanics and the theory behind the instantaneous center of rotation. An innovative and potentially effective method was presented to directly visualize the velocity vector field of simulated moving bodies. This approach could offer at least two advantages: (i) it can provide additional insight into the motion of bodies (translation, rotation, and general plane motion) compared to static images with a limited number of velocity vectors, and (ii) it can provide the opportunity to locate and visualize the instantaneous center of rotation in a straightforward manner. The mainly virtual workflow that was followed is based on algorithms used in particle image velocimetry, the current reference technique for measuring the instantaneous velocity field in flowing fluids. Although the method proved to be accurate, it is not put forward as a possible alternative to the existing procedures for solving problems found in standard mechanics textbooks. It is believed, however, that the visual component in the presented workflow may help learners to improve their overall understanding of rigid-body kinematics, and to relate quite abstract physics concepts to real situations. Perhaps writers of mechanics or physics textbooks could be encouraged to take up images showing the velocity field (instead of a discrete number of velocity vectors) to enhance the understanding of the kinematics of rigid bodies.

Finally, it is noted that the new approach was adopted here for two other reasons. First, if enough time is available in class, the instructor has the opportunity not only to show some of the velocity vector images, but also to explain the working principles of PIV to the students. We are convinced that the use of advanced research techniques (even if they are domain specific), such as PIV, will likely arouse the interest of first-year engineering and physics students in more advanced courses. And second, the results of this work might encourage other instructors to think about more innovative ways to visualize the motion of (virtual or physical) rigid bodies, or to come up with creative ideas to exploit the PIV technology outside its traditional application area.

\section{ACKNOWLEDGMENTS}

The author wishes to thank the unknown reviewers for their valuable advice and critical but helpful comments.

\section{APPENDIX: SOLUTIONS TO THE SLIDING ROD PROBLEM}

Problem: A rod of length $L=2 \mathrm{~m}$ slides against a wall as shown in Fig. 9. The velocity of point $A$ is given as $\vec{v}_{A}=$ $-5 \vec{j} \mathrm{~m} / \mathrm{s}$. Find the velocity of point $B$. 


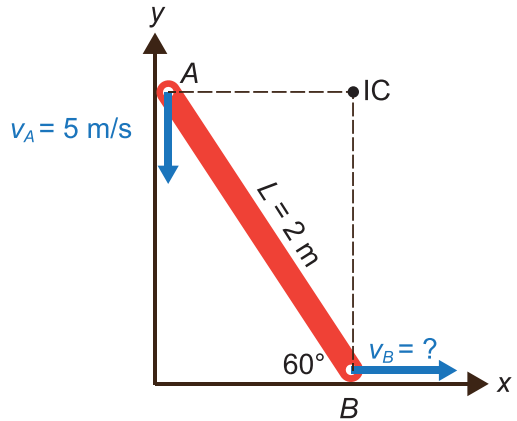

Fig. 9. An example of a typical introductory textbook problem on rigidbody kinematics.

Solution 1: Using the vector equation $\vec{v}_{B}=\vec{v}_{A}+\vec{\omega} \times \vec{r}_{B / A}$ Using Eq. (1), we can write $v_{B} \vec{i}=-5 \vec{j}+\omega \vec{k} \times\left(2 \cos 60^{\circ} \vec{i}\right.$ $\left.-2 \sin 60^{\circ} \vec{j}\right)$. The projection on the $x$-axis is $v_{B}=2 \omega \sin 60^{\circ}$, and the projection on the $y$-axis is $0=-5+2 \omega \cos 60^{\circ}$. The solution to this set of equation yields: $\omega=5 \mathrm{rad} / \mathrm{s}$ and $v_{B}=8.66 \mathrm{~m} / \mathrm{s}$.

Solution 2: Using the instantaneous center of rotation

The intersection of the two perpendiculars to $v_{A}$ and $v_{B}$ is the IC, which is located at $x=2 \cos 60^{\circ}, y=2 \sin 60^{\circ}$. Because the rod is instantaneously rotating about the IC, the well-known equations of circular motion $(v=\omega R$ or $\omega=v / R)$ can be used. We then find immediately that $\omega=$ $5 / 2 \cos 60^{\circ}=5 \mathrm{rad} / \mathrm{s}$ and $v_{B}=2 \omega \sin 60^{\circ}=8.66 \mathrm{~m} / \mathrm{s}$.

${ }^{1}$ F. P. Beer et al., Vector Mechanics for Engineers: Dynamics, 11th ed. (McGraw-Hill Higher Education, New York, 2015).

${ }^{2}$ R. C. Hibbeler, Engineering Mechanics: Dynamics, 14th ed. (Pearson, New York, 2016).

${ }^{3}$ J. L. Meriam, L. G. Kraige, and J. N. Bolton, Engineering Mechanics: Dynamics, 8th ed. (Wiley, New York, 2016).

${ }^{4}$ A. M. Bedford and W. Fowler, Engineering Mechanics: Dynamics, 5 th ed. (Pearson, New York, 2008)

${ }^{5}$ D. C. Giancoli, Physics for Scientists and Engineers with Modern Physics and MasteringPhysics. (Pearson Prentice Hall, New York, 2008).

${ }^{6} \mathrm{R}$. Serway and J. Jewett, Physics for Scientists and Engineers with Modern Physics, 9th ed. (Nelson Education, Toronto, Canada, 2016).

${ }^{7}$ R. Wolfson, Essential University Physics: Pearson New International Edition, 3rd ed. (Pearson, New York, 2016).

${ }^{8}$ S. Demircioglu, K. Yurumezoglu, and H. Isik, "Demonstrating the direction of angular velocity in circular motion," Phys. Teach. 53(6), 360-362 (2015).

${ }^{9} N X$ v10.0. Siemens PLM Software.

${ }^{10}$ Simwise 4D v9.5. Design Simulation Technologies.
${ }^{11}$ R. J. Adrian and J. Westerweel, Particle Image Velocimetry (Cambridge U.P., Cambridge, U.K., 2011).

${ }^{12}$ M. Raffel et al., Particle Image Velocimetry: A Practical Guide (SpringerVerlag, Berlin, Heidelberg, 2007).

${ }^{13}$ A. Schröder and C. E. Willert, Particle Image Velocimetry: New Developments and Recent Applications (Springer, Berlin Heidelberg, 2008).

${ }^{14}$ C. E. Willert and M. Gharib, "Digital particle image velocimetry," Expts. Fluids 10(4), 181-193 (1991).

${ }^{15} \mathrm{~W}$. Thielicke and E. Stamhuis, "PIVlab-Towards user-friendly, affordable and accurate digital particle image velocimetry in MATLAB," J. Open Res. Softw. 2(1), 1-10 (2014).

${ }^{16}$ Matlab R2015. Mathworks.

${ }^{17}$ A. De Ambrosis et al., "Investigating the role of sliding friction in rolling motion: a teaching sequence based on experiments and simulations," Euro. J. Phys. 36(3), 035020 (2015).

${ }^{18}$ M.-L. López, "Angular and linear acceleration in a rigid rolling body: students' misconceptions,” Euro. J. Phys. 24(6), 553-562 (2003).

${ }^{19}$ L. G. Rimoldini and C. Singh, "Student understanding of rotational and rolling motion concepts," Phys. Rev. Phys. Ed. Res. 1(1), 010102 (2005).

${ }^{20} \mathrm{~W}$. Anderst et al., "Motion path of the instant center of rotation in the cervical spine during in vivo dynamic flexion-extension implications for artificial disc design and evaluation of motion quality after arthrodesis," Spine 38(10), E594-E601 (2013).

${ }^{21}$ E. Baillargeon and W. J. Anderst, "Sensitivity, reliability and accuracy of the instant center of rotation calculation in the cervical spine during in vivo dynamic flexion-extension," J. Biomech. 46(4), 670-676 (2013).

${ }^{22}$ J. H. Hollman et al., "Gender differences in surface rolling and gliding kinematics of the knee," Clin. Ortho. Rel. R. 413, 208-221 (2003).

${ }^{23}$ J. D. Moorehead, S. C. Montgomery, and D. M. Harvey, "Instant center of rotation estimation using the Reuleaux technique and a Lateral Extrapolation technique," J. Biomech. 36(9), 1301-1307 (2003).

${ }^{24} \mathrm{~S}$. R. Thomas et al., "Geometrical analysis of Copeland surface replacement shoulder arthroplasty in relation to normal anatomy," J. Shoulder Elbow Surg. 14(2), 186-192 (2005).

${ }^{25} \mathrm{G}$. R. Pennock and A. Israr, "Kinematic analysis and synthesis of an adjustable six-bar linkage," Mech. Mach. The. 44(2), 306-323 (2009).

${ }^{26}$ J. K. Lee and J. K. Shim, "Validity and limitations of the kinematic roll center concept from the viewpoint of spatial kinematics using screw theory," Int. J. Automot. Technol. 12(5), 769 (2011).

${ }^{27}$ D. Weagle, "Vehicle suspension systems," Google Patents No. US 7128329 B2 (2006)

${ }^{28}$ J. T. Whitney, "Reactive steering, instant center, twin linkage system for use with tag trailers for improved towability and safety," Google Patents No. US 20140151978 A1 (2014).

${ }^{29}$ S. P. Arnoczky, P. A. Torzilli, and J. L. Marshall, "Biomechanical evaluation of anterior cruciate ligament repair in dog - analysis of instant center of motion," J. Am. Anim. Hosp. Assoc. 13(5), 553-558 (1977).

${ }^{30}$ Algodoo v2.1. Algoryx. See http://www.algodoo.com.

${ }^{31} \mathrm{~S}$. Annerel et al., "Validation of a numerical FSI simulation of an aortic BMHV by in vitro PIV experiments," Med. Eng. Phys. 36(8), 1014-1023 (2014)

${ }^{32}$ SecondSightDigital, "Knee centrodes" at https://www.youtube.com/ watch?v=UtdSJZn62H8 (2012). 